all samples analysed. T. pallidum subtyping discriminated reinfection.

\section{P1.45 EVALUATION OF SEKURE RPR REAGENT ON THE SK500 CLINICAL CHEMISTRY SYSTEM}

Kara Osbak, S Abdellati, A Tsoumanis, M Van Esbroeck, T Crucitti, C Kenyon. Institute of Tropical Medicine, Antwerp, Belgium

\subsection{6/sextrans-2017-053264.152}

Introduction An automated and accurate laboratory assay would be of considerable utility to the diagnosis of syphilis and treatment follow-up. We compared the Sekure RPR (Rapid Plasma Reagin) test performed on the SK500 Clinical Chemistry System to RPR card test results.

Methods Serum samples were collected in the context of a 2 year observational cohort study of syphilis infected patients and controls. Syphilis was diagnosed using non-treponemal and treponemal testing. Sera collected at the time of diagnosis (M0) and at 3, 6, 9 and 12 months post-treatment were tested by a Macro-Vue RPR card test (RPR-C) (Becton Dickinson) and a Sekure RPR test (RPR-S) (Sekisui Diagnostics). RPR-S results are expressed in RPR units (R.U.), whereby 1 R.U. equals a 1 -fold change in RPR-C titre. The agreement, linearity and reportable ranges were determined using RPR-C results as the gold standard. Linear regression was used to assess correlations from before and after implementation of an extra dilution step for samples with a strong suspicion of prozone effect.

Results In total, 451 samples from 150 participants were tested, including 120 new syphilis cases and 30 controls. All 30 controls tested negative. Initially there was a weak correlation between RPR-C and RPR-S values $(r=0.15)$. Further analyses identified 72 RPR-S samples with a strong suspicion of prozone effect. We therefore included an extra dilution step (10x) and retested 60/72 samples; values within the expected range were obtained for 58 of them. After implementing the extra dilution step the correlation was moderate $(r=0.61)$, increasing further to $\mathrm{r}=0.91$ for samples with RPR-C titres $\leq 128$. Of the 92 samples that tested RPR-C positive and RPR-S negative, 8 were from M0 (RPR-C: $1-4$ ), which would have led to missed diagnoses.

Conclusion A reasonable correlation was found between the tested methods for mid-range RPR-C results (titre $\leq 128$ ). However, prozone may occur in samples with high antibody concentrations. More investigation is required to elucidate the false negative RPR-S results.

\section{P1.46 COMPARISON OF TWO ENZYME IMMUNOASSAYS FOR THE DETECTION OF IGG AND IGM ANTI-TREPONEMA PALLIDUM ANTIBODIES}

Kara Osbak, S Abdellati, A Tsoumanis, M Van Esbroeck, T Crucitti, C Kenyon. Institute of Tropical Medicine, Antwerp, Belgium

\subsection{6/sextrans-2017-053264.153}

Introduction We aimed to compare two commercial enzyme immunoassays (EIA) for the detection of IgG and IgM antiTreponema pallidum (Tp) antibodies.

Methods Serum samples were collected in the context of a larger study looking at diagnostic biomarkers for syphilis.
Patients with syphilis diagnosed by a treponemal and a nontreponemal assay, were followed for up to two years after treatment. Specimens collected at visit of diagnosis (B), and after three (M3) and six months (M6) of treatment were tested by EIAs detecting anti-Tp IgG and IgM from the manufacturers Euroimmun (EU) and Mikrogen (MI).

Results We tested 338 samples collected from 119 new syphilis cases (23 primary (P), 49 secondary (S), 31 early latent (EL), 16 late latent (LL)) and 30 uninfected controls. A total of 40 participants contributed to 1 sample, 29 to 2 samples and 80 to 3 samples. The controls contributed only to the samples collected at B. Overall 147, 86 and 105 samples were obtained at B, M3 and M6, respectively. The IgM assays were in agreement for $78,1 \%$ of samples; it varied according to the syphilis stage: P: $82,1 \%$; S: $72,5 \%$; EL: $80,8 \%$; LL: $72,1 \%$ and decreased from B to M3: B: 84,4\%; M3: 73,3\%; M6: $73,3 \%$. More samples tested positive with the MI (149) versus the EU (100) $(\mathrm{p}<0.001)$. EU tested all control samples IgM negative, MI reported 1 positive and 1 borderline. The agreement of both $\operatorname{IgG}$ assays was $97,4 \%$; it increased with the stage of infection: P: 91,1\%; S: 97,7\%; EL: 100\%; LL: 100\%, and over time: B: 95,2\%: M3: 98,8\%; M6: 99,0\%. More samples tested positive with the EU (305) versus the MI (300) assay. EU reported all control samples IgG negative, MI detected 1 borderline sample.

Conclusion A good but not perfect agreement was observed for the EIAs detecting IgM. The agreement was highest in primary syphilis and lowest in late latent cases, and decreased over time of treatment. The MI IgM assay reported significantly more positive samples. Overall, we found a good agreement for the EIAs detecting IgG. Albeit that it was somewhat lower for primary syphilis and at baseline.

\section{P1.47 DETECTION OF CERVICAL HUMAN PAPILLOMAVIRUS IN WOMEN ATTENDING FOR CERVICAL CANCER SCRENNING BY VISUAL INSPECTION IN COTE D'IVOIRE}

${ }^{1}$ Ouattara Abdoulaye, ${ }^{2}$ Yeo Alain, ${ }^{2}$ Kouame-Blavo Ehui, ${ }^{3}$ Oura K Pierre, ${ }^{2}$ FayeKette Hortense, ${ }^{2}$ Dosso Mireille. ${ }^{1}$ Felix Houphouet Boigny University, Abidjan - Cote D Ivoire; ${ }^{2}$ Institut Pasteur Cote D'ivoire, Abidjan - Cote D Ivoire; ${ }^{3}$ Hopital General D'abobo Sud Service Gynecoielog, Abidjan - Cote D Ivoire

\subsection{6/sextrans-2017-053264.154}

Introduction Human Papillomaviruses (HPV) are small virus non-enveloped double-stranded circular DNA responsible of genital warts papilloma, precancerous lesions and cancers (cervix, vulva). In Côte d'Ivoire and many lower middle and incomes countries cervical cancer screening program based on visual inspection methods become the gold standard because cytology has shown many limits. This study aims to detect HPV DNA on women attending for cervical cancer screening program based on visual inspection by acid acetic and lugol (IVA/IVL).

Methods From March to December 2015, endocervical secretions from women attending cervical screening by IVA were submitted to HPV determination with PCR. HPV DNA was amplified using PGMY09/11 primers which generated 450 base pairs at the L1 region. The samples harbouring HPV DNA were genotyped using the multiplex PCR with HPV 16, $18,31,33,35,45$ and 51 primers.

Results The medium age of population was 32 years old. On 388 women enrolled in a visual inspection with acetic acid (VIA) program 5.8\% were positif. HPV DNA was obtained in 